\title{
Expression and Characterization of Functional Recombinant Bet v 1.0101 in the Chloroplast of Chlamydomonas reinhardtii
}

\author{
Sonja Hirschl ${ }^{a}$ Claudia Ralser $^{a}$ Claudia Asam $^{b}$ Alessandro Gangitano ${ }^{a}$ \\ Sara Huber $^{b}$ Christof Ebner ${ }^{c}$ Barbara Bohle ${ }^{d}$ Martin Wolf ${ }^{b}$ Peter Briza ${ }^{b}$ \\ Fatima Ferreira $^{\mathrm{b}}$ Christoph Griesbeck ${ }^{\mathrm{a}}$ Michael Wallner $^{\mathrm{b}}$ \\ ${ }^{a}$ Department of Biotechnology and Food Engineering, MCI Management Center Innsbruck, Innsbruck, \\ ${ }^{b}$ Department of Molecular Biology, University of Salzburg, Salzburg, and ' Allergy Clinic Reumannplatz and \\ ${ }^{\mathrm{d}}$ Department of Pathophysiology and Allergy Research and Christian Doppler Laboratory for Immunomodulation, \\ Medical University of Vienna, Vienna, Austria
}

\section{Keywords}

Chlamydomonas reinhardtii · Bet $\mathrm{v} 1$ - Allergy vaccine .

Recombinant allergen

\begin{abstract}
Background: Allergen immunotherapy (AIT) still plays a minor role in the treatment of allergic diseases. To improve the acceptance of AIT by allergic patients, the treatment has to become more convenient and efficacious. One possibility is the oral application of allergens or derivatives thereof. Therefore, we sought to produce a recombinant allergen in the green alga Chlamydomonas reinhardtii as a novel production platform. Methods: The major birch pollen allergen Bet $v 1$ was selected as candidate molecule, and a codon-optimized gene was synthesized and stably integrated into the microalga C. reinhardtii FUD50. Positive
\end{abstract}

Sonja Hirschl, Claudia Ralser, and Claudia Asam contributed equally to the study.

\section{KARGER}

E-Mail karger@karger.com www.karger.com/iaa

\section{(C) 2017 The Author(s)}

Published by S. Karger AG, Basel

Karger

Open access

This article is licensed under the Creative Commons AttributionNonCommercial-NoDerivatives 4.0 International License (CC BYNC-ND) (http://www.karger.com/Services/OpenAccessLicense) Usage and distribution for commercial purposes as well as any distribution of modified material requires written permission. transformants were identified by PCR, cultured, and thereafter cells were disrupted by sonication. Bet $v 1$ was purified from algal total soluble protein (TSP) by affinity chromatography and characterized physicochemically as well as immunologically. Results: All transformants showed expression of the allergen with yields between 0.01 and $0.04 \%$ of TSP. Algal-derived Bet $v 1$ displayed similar secondary structure elements as the Escherichia coli-produced reference allergen. Moreover, Bet v 1 produced in C. reinhardtii showed binding comparable to human $\lg E$ as well as murine Bet v 1-specific IgG. Conclusion: We could successfully produce recombinant Bet $v 1$ in C. reinhardtii. As microalgae are classified as GRAS (generally recognized as safe), the pilot study supports the development of novel allergy treatment concepts such as the oral administration of allergen-containing algal extracts for therapy.

(c) 2017 The Author(s)

Published by S. Karger AG, Basel

Correspondence to: Dr. Michael Wallner

Department of Molecular Biology, University of Salzburg Hellbrunnerstreet 34

AT-5020 Salzburg (Austria)

E-Mail michael.wallner@sbg.ac.at

Dr. Christoph Griesbeck

Department of Biotechnology and Food Engineering, MCI

Maximilianstreet 2

AT-6020 Innsbruck (Austria)

E-Mail Christoph.griesbeck@mci.edu 


\section{Introduction}

Worldwide, the prevalence of allergic diseases is still increasing. Treatment guidelines for allergies include strategies to avoid allergen exposure, anti-inflammatory medication, as well as allergen immunotherapy (AIT), whereas the latter is the only potentially diseasemodifying treatment option [1]. The basic principle of AIT comprises the repeated administration of diseaseeliciting allergens, which eventually induces a modulation of the allergic immune response. Mechanisms of AIT include early desensitization of effector cells, the induction of anti-inflammatory $\mathrm{T}$ as well as $\mathrm{B}$ cells, and the modulation of the allergen-specific IgE responses, including the induction of blocking antibodies [2]. Recombinant allergens were shown to be safe and efficacious alternatives to allergen extracts, which are widely applied in AIT [3]. However, recombinant production of allergens still needs improvement. The unicellular green alga Chlamydomonas reinhardtii offers the possibility to express proteins in the nucleus as well as in the chloroplast, where higher expression rates were reported, but no posttranslational modifications are possible since the proteins stay in the chloroplast $[4,5]$. Microalgae such as $C$. reinhardtii have been acknowledged as GRAS (generally recognized as safe) production platforms [6] for therapeutic proteins, implying that algal products are essentially free of human pathogens. In contrast, the outer membrane of Escherichia coli contains the potent immunostimulatory molecule lipopolysaccharide, which has to be removed cost intensively after the production of therapeutic proteins in these cells [7]. Recombinant protein production in $C$. reinhardtii is scalable, and low production costs may be achieved. Moreover, algal-derived edible allergy vaccines could be envisioned using this expression platform. Compared to yeast systems, relatively accurate posttranslational modifications, i.e., glycosylation patterns, may be achieved [8]. Therefore, we sought to explore this expression system for the recombinant production of allergen products using the major birch pollen allergen Bet $\mathrm{v} 1$ as a model. The production process was complemented by a comprehensive comparison of algal-derived Bet $\mathrm{v} 1$ with a batch of recombinant Bet $\mathrm{v}$ 1 produced in E. coli [9]. Previously, careful comparisons of natural Bet $\mathrm{v} 1$ with Bet v 1 produced in E. coli confirmed that the bacterial-derived allergen can be used as a surrogate for the natural protein. Thus, E. coli Bet $\mathrm{v} 1$ was used as reference material in the study [9, $10]$.

Chlamydomonas-Derived Bet v 1

\section{Materials and Methods}

\section{Patients and Sera}

Birch pollen-allergic $(n=25)$ and house dust mite-allergic individuals $(n=10)$, and nonallergic donors $(n=5)$, were selected based on case history, in vivo skin prick testing; and in vitro IgE detection (CAP system, Thermo Fisher Scientific, Phadia AB). Experiments with patients' sera were approved by the Ethics Committee of the University of Vienna (EK028/2006), and informed consent was obtained from all subjects included in the study.

\section{Cultivation of Algal Strains}

The C. reinhardtii atpB deletion mutant CC-1185 mt+ (FUD50) [11] was cultured heterotrophically in Tris-acetate-phosphate medium [12], and transformants were grown in high-salt medium (HSM) [12] without acetate supplemented with ampicillin $(50 \mu \mathrm{g} /$ $\mathrm{mL}$ ). Liquid cultures were performed under continuous light (50 $\mu \mathrm{mol} / \mathrm{m}^{-2} \times \mathrm{s}^{-1}$ ) at $25^{\circ} \mathrm{C}$.

\section{Construction of the Transformation Vector atpB int}

(psaA::bet $v 1$ ) and Transformation

The birch pollen allergen sequence bet $v 1.0101$ was codon optimizedfor C. reinhardtii, produced syntheticallybyATG:biosynthetics $\mathrm{GmbH}$ (Regensburg) and cloned into the vector atpBint (psaA::aadA) via the restriction sites $S p h \mathrm{I}$ and $\mathrm{NcoI}$. The obtained plasmid atpB int (psaA::bet v 1) (Fig. 1) was transformed into the chloroplast of $C$. reinhardtii FUD50 via particle bombardment (gold, $0.6 \mu \mathrm{m}$ ). Recombinant clones were selected by growth on plates with minimal medium (HSM plates [13] under continuous light [50 $\mu \mathrm{mol} /$ $\left.\mathrm{m}^{-2} \times \mathrm{s}^{-1}\right]$ ) at room temperature. To achieve homoplasmic chloroplasts, cells were spread several times onto fresh HSM agar.

\section{Protein Analysis}

Pellets of $3 \times 10^{7}$ cells were washed $(1 \times$ PBS $+0.025 \%$ Tween $20)$ and resuspended in $30 \mu \mathrm{L}$ lysis buffer $(30 \mathrm{mM}$ Tris $\mathrm{HCl}, \mathrm{pH} 8$, $15 \%$ sucrose, cOmplete ${ }^{\mathrm{TM}}$ protease inhibitor) for Western blot and ELISA, or in $50 \mathrm{mM} \mathrm{NaPO}_{4}$ buffer ( $\mathrm{pH}$ 7.4) for protein purification, and lysed by sonication. Total soluble protein (TSP) of the supernatant was determined with the $\mathrm{DC}^{\mathrm{TM}}$ protein assay (BioRad).

Immunoblots were performed with BIP-1 primary antibody (1 $\mu \mathrm{g} / \mathrm{mL}$ ) [14] to detect Bet 1.0101 or anti-RbcL (AS03 037, Agrisera) as positive control and an alkaline phosphatase-conjugated goat anti-rabbit IgG (Jackson ImmunoResearch Laboratories, Inc., West Grove, PA, USA) secondary antibody (AS09 607, Agrisera). ELISA was performed as described [9].

\section{Purification of Bet $v 1$}

Algal-derived Bet v 1.0101 (termed Bet v 1 Cr thereafter) was purified from TSP using the monoclonal mouse anti-Bet v 1 IgG BIP-1 coupled to Sepharose activated by normal human sera (GE Healthcare, Chalfont St. Giles, UK) [14]. Reference Bet v 1.0101 (termed Bet v 1 Ec) was produced as described [14]. Recombinant proteins were dialyzed against $10 \mathrm{mM}$ sodium phosphate buffer and stored at $-20^{\circ} \mathrm{C}$. Purity was analyzed by SDS-PAGE, and protein identity by mass spectrometry using a Q-Exactive LC-MS/MS system (Thermo Fisher Scientific).

\section{Physicochemical Characterization}

Secondary structure analysis of recombinant proteins was performed by circular dichroism spectroscopy using a Jasco J-815

Int Arch Allergy Immunol 2017;173:44-50 


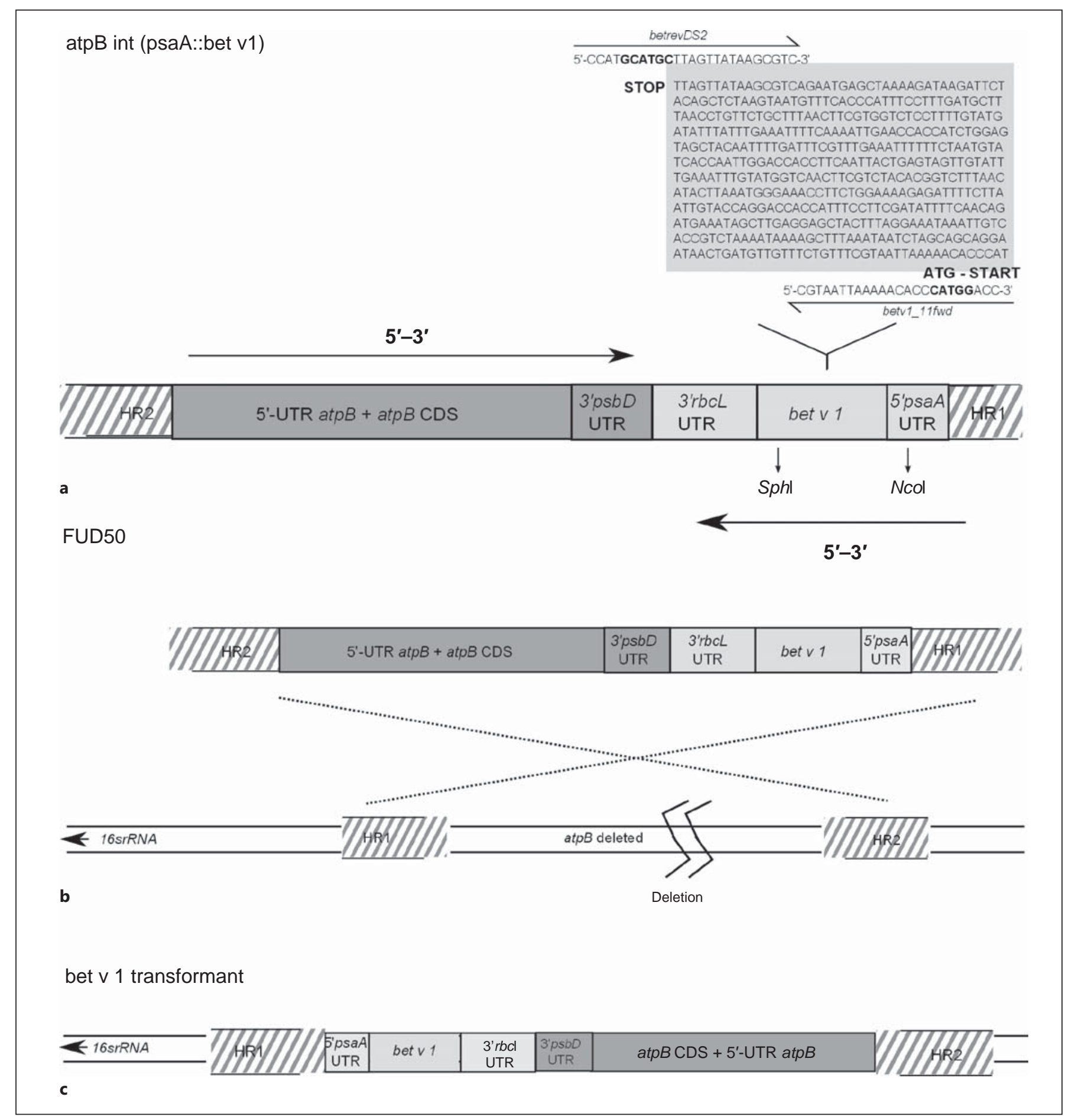

Fig. 1. Scheme of the chloroplast expression vector atpB int (psaA::bet v 1), the integration locus and the plastid genome of the transformant. a atpB int (psaA::bet v 1), containing the transgene expression cassette (gray) and the atpB restoration cassette (dark gray). The gene of interest bet $v 1.0101$ (gray) is flanked by the untranslated regions (UTR) of $C$. reinhardtii chloroplast genes $5^{\prime}$ psaA and $3^{\prime}$ rbcL. Restriction sites (SphI and NcoI) and primers (betrevDS2, betv1_11fwd with restriction sites in bold) for cloning are indicated, the codon-optimized antisense bet $v 1.0101$ sequence is depicted. b In FUD50, atpB is deleted. The ability to grow photoautotrophically is restored due to the integration of atpB (dark gray) from the expression vector atpB int (psaA::bet v 1). Both expression cassettes, bet $v 1.0101$ and atpB, are integrated into the plastid genome via homologous recombination, between HR1 and HR2 (hatched). c Resulting transformant genome. 
Fig. 2. Total soluble proteins of untransformed $C$. reinhardtii strain FUD50 and transformants T30.1.2, T30.1.3, and T32.3.1 were extracted and examined in a Western blot for the presence of the 52.3$\mathrm{kDa}$ chloroplast housekeeping protein $\mathrm{RbcL}$ (a) and the 17.4-kDa Bet v 1.0101 (b).

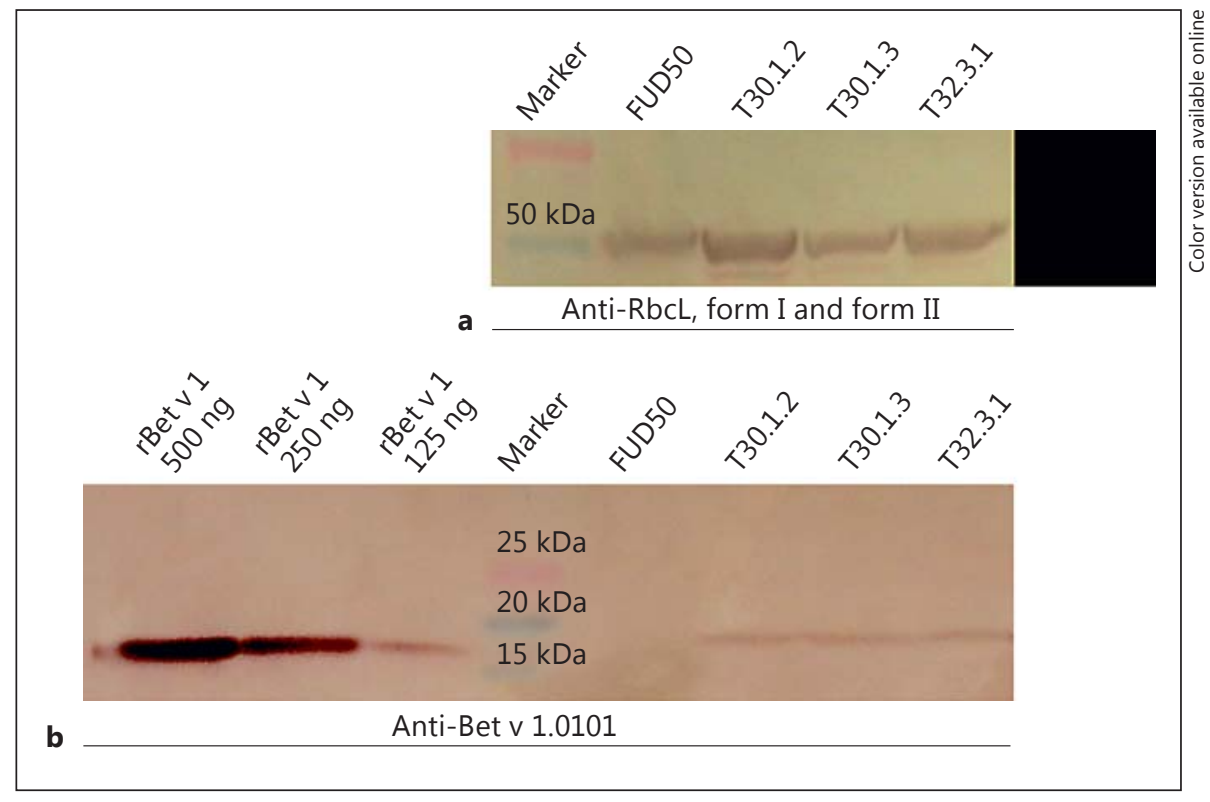

spectropolarimeter fitted with a PTC-423S Peltier-type singleposition cell holder (Jasco, Tokyo, Japan) [14]. Accessibility and functionality of the intrinsic cavity was determined by 1 -anilino8-naphthalene sulfonate (ANS) displacement assays [14].

\section{Immunological Characterization}

For ELISA experiments, plates were coated with allergen (200 $\mathrm{ng} /$ well in $50 \mu \mathrm{L} \mathrm{PBS}$ ) overnight at $4^{\circ} \mathrm{C}$. After blocking, plates were incubated with either patients' sera diluted 1:10 or serial dilutions of monoclonal mouse anti-Bet v 1 IgG antibodies $[14,15]$ starting at a concentration of $1 \mu \mathrm{g} / \mathrm{mL}$ overnight at $4^{\circ} \mathrm{C}$. Bound human IgE as well as murine $\mathrm{IgG}$ was detected with alkaline phosphatase-conjugated monoclonal anti-human IgE antibodies (BD Biosciences, Franklin Lakes, NJ, USA) or alkaline phosphatase-conjugated rat anti-mouse IgG1 (Southern Biotech, Birmingham, AL, USA), respectively. For ELISA inhibitions, plates were coated with $2 \mu \mathrm{g} / \mathrm{mL}$ birch pollen extract. A serum pool of 10 birch pollen-allergic patients monosensitized to Bet $\mathrm{v} 1$ was diluted $1 / 20$ and incubated with serial dilutions of either Bet $\mathrm{v} 1 \mathrm{Ec}$ or Bet $\mathrm{v} 1 \mathrm{Cr}$ overnight at $4^{\circ} \mathrm{C}$. After washing and blocking, the inhibited serum pool was added to the coated ELISA plate for $3 \mathrm{~h}$ at room temperature. Bound IgE was detected as described above. IgE measurements were performed in duplicates. For human IgE binding, statistics were calculated with paired-sample $t$ test, whereas for murine monoclonal antibodies statistics were calculated with repeatedmeasure ANOVA using the Tukey test for multiple comparisons. $p$ values $<0.05$ were considered significant.

\section{Results}

Cloning and Transformation of atpB int (psaA::bet $v 1$ )

Recombinant Bet v 1 Cr was derived by cloning the codon-optimized bet $v 1.0101$ (Fig. 1a-c) into the expres- sion vector atpB int (psaA::bet v 1). As natural Bet v 1 is a nonglycosylated protein, Bet v $1 \mathrm{Cr}$ was produced in the chloroplast of $C$. reinhardtii where no protein glycosylation occurs [16]. Chloroplasts of $C$. reinhardtii strain FUD50 cells (atpB deletion) were transformed by particle bombardment, and positive transformants were selected by restoration of photoautotrophy on HSM plates. Homoplasmicity for the gene of interest was tested after several passages (data not shown).

\section{Bet $v 1$ Expression in $C$. reinhardtii}

$3 \times 10^{7}$ cells of transformants T30.1.2, T30.1.3, T32.3.1 3 , as well as untransformed FUD50 cells were harvested and lysed by sonication. TSP was isolated and used for Western blotting (Fig. 2). Bet v 1 was detected in all transformants with yields of approximately $125 \mathrm{ng}(0.125 \%)$ per $100 \mu \mathrm{g}$ TSP. This was verified using a Bet v 1-specific sandwich ELISA where Bet v 1 concentrations were between 0.01 and $0.04 \%$ of TSP.

\section{Bet $v 1$ Cr Showed Similar Structural Properties as \\ Bet $v 1 \mathrm{Ec}$}

Bet v 1 Cr was purified from the soluble fraction of $C$. reinhardtii lysates by antibody affinity chromatography, and purity was confirmed by SDS-PAGE (Fig. 3a). Massspectrometric analysis indicated that Bet v $1 \mathrm{Cr}$ was not modified during the translation process in the algal chloroplast or during protein purification (theoretical monoisotopic mass $=17,428.93 \mathrm{Da}$, experimentally determined monoisotopic mass $=17,428.88 \mathrm{Da}, \Delta \mathrm{m}=0.05)$. 


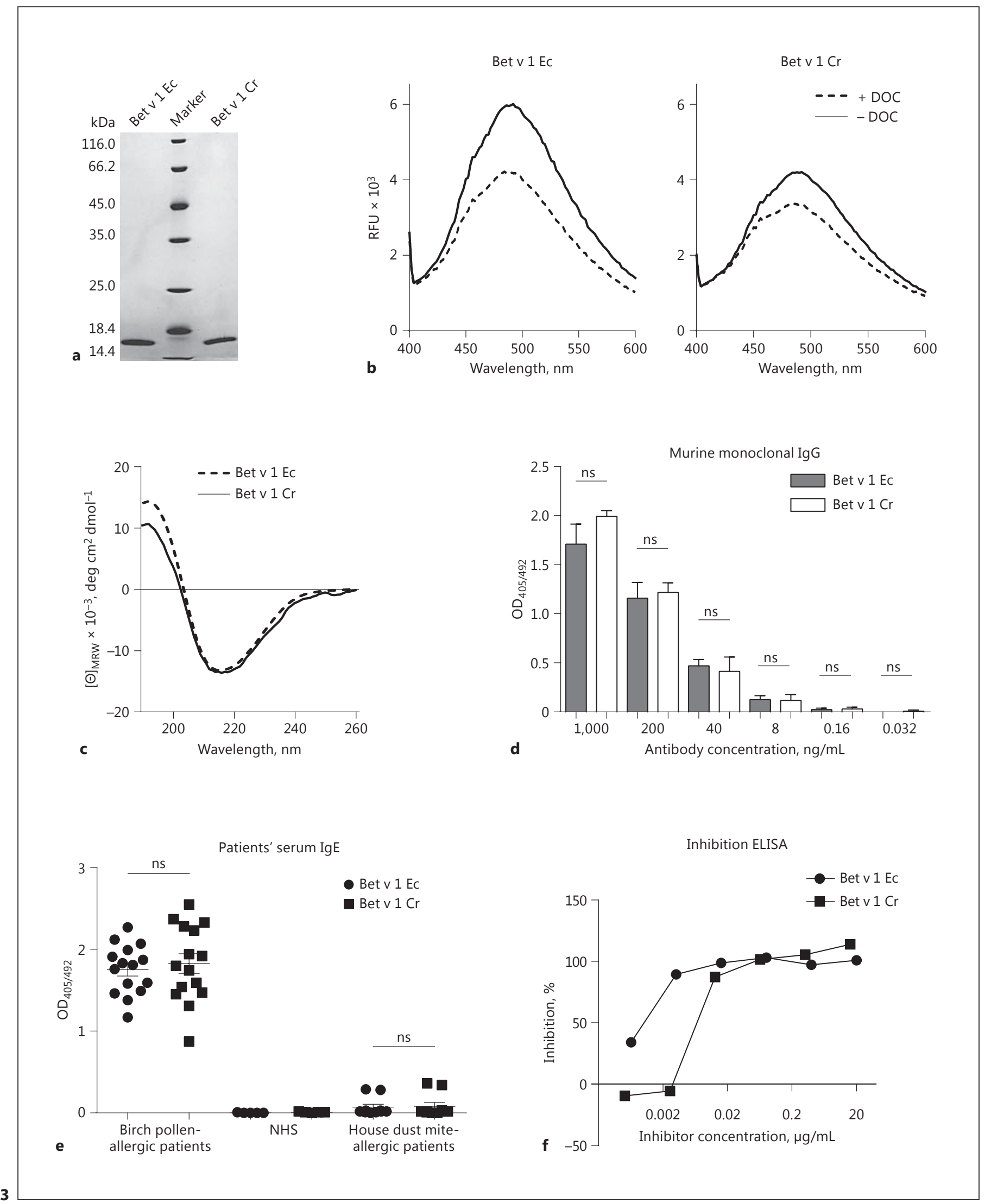

(For legend see next page.) 
Bet $\mathrm{v} 1$ harbors an intrinsic hydrophobic cavity able to bind the sulfonic acid ANS. This binding can be blocked by addition of sodium deoxycholate (DOC). Therefore, ANS displacement assays were performed to investigate solvent accessibility of the Bet $\mathrm{v} 1$ cavity. We found that both Bet v 1 Ec and Cr could bind ANS, whereas the addition of DOC in a 1:2 molar ratio reduced ANS binding in both allergen preparations (Fig. 3b). Circular dichroism spectra were recorded to compare secondary structure elements of Bet v 1 batches. Analyses of the proteins resulted in essentially the same spectra, both in amplitudes as well as curve shape, indicating similar secondary structures (Fig. 3c).

\section{Antibody Binding Properties of Bet $v 1 \mathrm{Cr}$}

To compare the antibody binding properties of Bet $\mathrm{v} 1$ $\mathrm{Cr}$ and Ec, a panel of 4 murine monoclonal Bet v 1-specific antibodies was used (Fig. 3d). The antibodies were titrated; however, we could not find significant differences in antibody binding to any Bet $\mathrm{v} 1$ batch. Thereafter, human serum IgE binding to Bet v $1 \mathrm{Cr}$ and $\mathrm{Ec}$, respectively, was determined using sera of Bet v 1-allergic patients $(n=15)$, normal human sera $(n=5)$, as well as sera of house dust mite-allergic donors $(n=10)$ (Fig. 3e). Again, we did not find differences in IgE reactivity profiles between E. coli- and C. reinhardtii-derived Bet v 1 . Finally, we performed inhibition ELISA using a serum pool of 10 birch pollen-allergic patients monosensitized to Bet v 1 (Fig. 3f). Here, Bet v 1 Ec was a stronger inhibitor of serum IgE than algal-derived Bet $\mathrm{v} 1$.

\section{Discussion}

When looking at the landscape of protein-based pharmaceuticals, glycoproteins represent the largest section of biologically derived drugs approved by the European Medicines Agency, whereas $\mathrm{CHO}$ (Chinese hamster ovary) cells are the most widely used production platform. Nonglycosylated therapeutic proteins are typically pro-

Fig. 3. a SDS-PAGE confirming purity of Bet v $1 \mathrm{Ec}$ as well as $\mathrm{Cr}$ batches. $\mathbf{b}$ Competitive binding of ANS and DOC to Bet $\mathrm{v} 1$ batches was analyzed by fluorescence spectroscopy; data are baseline corrected. c Circular dichroism spectra were recorded for the determination of secondary structure elements; data are baseline corrected and presented as mean residue molar ellipticity. $\mathbf{d}$ Antibody binding of purified murine monoclonal antibodies $(n=4)$ to Bet $\mathrm{v} 1$ batches was tested by ELISA. Statistical analyses were per-

Chlamydomonas-Derived Bet v 1 duced in E. coli followed by Saccharomyces cerevisiae, whereas most approved vaccines are being produced in hen's eggs [17]. In recent years, microalgae have been postulated as a possible alternative to established production platforms for industrial-scale production of recombinant pharmaceuticals. The advantages of algal-based systems depend primarily on the algal strain; in general, expression systems based on microalgae offer high protein folding accuracy, sufficient yields, and scalable processes to affordable costs [8]. Probably one of the biggest advantages is that many microalgae are classified as GRAS [4] (www.fda.gov). So far, C. reinhardtii has been successfully used to express antigens from various pathogens as vaccine candidates [18], and very recently the major peanut allergens Ara h 2 as well as a truncated version of Ara h 1 have been produced using C. reinhardtii as expression platform [19].

In this study, we generated for the first time stable transformants of $C$. reinhardtii expressing an inhalant allergen, namely Bet $\mathrm{v} 1$. A codon-optimized gene (bet $v$ 1.0101; Fig. 1a) was inserted into the expression vector atpB int (psaA::bet v 1) [11]. The system has the advantage that selection via photoautotrophy is possible, and hence no antibiotics are needed. We ended up with a transgene expression of $0.01-0.04 \%$ of TSP compared to $0.8 \%$ TSP using this vector and up to $5 \%$ of TSP of recombinant protein produced in the chloroplast of C. reinhardtii reported by others [8]. Therefore, further studies have to be performed to enhance the expression rate. After protein purification, detailed physicochemical comparison of algal-derived Bet $\mathrm{v} 1$ with an E. coli-produced batch did not reveal any differences. Similar findings were obtained from the immunological characterization, where both batches of Bet $\mathrm{v} 1$ showed comparable antibody binding profiles with murine monoclonal antibodies as well as IgE antibodies derived from patients' sera. Interestingly, in inhibition ELISA, Bet $\mathrm{v} 1 \mathrm{Ec}$ was a stronger inhibitor of serum IgE than Bet v 1 Cr. Nevertheless, our results suggest that algal-derived Bet v 1 has similar immunologic properties as its E. coli-produced counter-

formed with repeated-measure ANOVA. e IgE binding of birch pollen-allergic patients' sera $(n=15)$, normal human sera (NHS) $(n=5)$, as well as house dust mite-allergic patient sera $(n=10)$ to Bet v 1 Ec or Bet v 1 Cr, respectively, was tested by ELISA. Statistical analyses were performed with Paired-sample $t$ tests. f For inhibition ELISA, birch pollen extract was coated onto a microtiter plate and human serum IgE binding (pool of 10 birch pollen-allergic patients' sera) was inhibited by either Bet v 1 Ec or Bet v 1 Cr.

Int Arch Allergy Immunol 2017;173:44-50 DOI: $10.1159 / 000471852$ 
part. This pilot study now offers novel strategic concepts for AIT using algal-derived allergen products. It is probably not necessary to purify the allergens from algal extracts, but AIT formulations based on allergen-containing algal preparations applicable for sublingual or oral immunotherapy seem possible. At the moment, we are at the forefront of researching algal-derived allergen products. Further studies are mandatory to evolve such concepts; however, we strongly believe in the high potential of algal-derived biologics.

\section{Acknowledgements}

The study was supported by the priority program "AllergyCancer-BioNano Research Centre" of the University of Salzburg, the Austrian Research Funds (FWF) P26125, and the TWF (Tiroler Wissenschaftsfonds, GZ: UNI-0404/1444).

C. reinhardtii atpB deletion mutant CC-1185 mt+ (FUD50) and the plasmid atpB int (psaA::aadA) were kindly provided by Michel Goldschmidt (Clermont, University of Geneva).

\section{Disclosure Statement}

All authors declare that in relation to this publication there is no conflict of interest.

\section{References}

1 WAO White Book on Allergy. Milwaukee, World Allergy Organization, 2011.

2 Akdis M, Akdis CA: Mechanisms of allergenspecific immunotherapy: multiple suppressor factors at work in immune tolerance to allergens. J Allergy Clin Immunol 2014;133:621631.

3 Ferreira F, Wolf M, Wallner M: Molecular approach to allergy diagnosis and therapy. Yonsei Med J 2014;55:839-852.

4 Griesbeck C, Kobl I, Heitzer M: Chlamydomonas reinhardtii: a protein expression system for pharmaceutical and biotechnological proteins. Mol Biotechnol 2006;34:213-223.

5 Harris EH: Chlamydomonas as a model organism. Annu Rev Plant Physiol Plant Mol Biol 2001;52:363-406.

6 Rasala BA, Mayfield SP: Photosynthetic biomanufacturing in green algae; production of recombinant proteins for industrial, nutritional, and medical uses. Photosynth Res 2015; 123:227-239.

7 Mamat U, Wilke K, Bramhill D, Schromm AB, Lindner B, Kohl TA, Corchero JL, Villaverde A, Schaffer L, Head SR, Souvignier C, Meredith TC, Woodard RW: Detoxifying Escherichia coli for endotoxin-free production of recombinant proteins. Microb Cell Fact 2015;14:57.
8 Yan N, Fan C, Chen Y, Hu Z: The potential for microalgae as bioreactors to produce pharmaceuticals. Int J Mol Sci 2016;17:E962.

9 Wallner M, Hauser M, Himly M, Zaborsky N, Mutschlechner S, Harrer A, Asam C, Pichler U, van Ree R, Briza P, Thalhamer J, Bohle B, Achatz G, Ferreira F: Reshaping the Bet v 1 fold modulates $\mathrm{T}(\mathrm{H})$ polarization. J Allergy Clin Immunol 2011;127:1571.e9-1578.e9.

10 Himly M, Nony E, Chabre H, Van Overtvelt L, Neubauer A, van Ree R, Buchheit KH, Vieths S, Moingeon P, Ferreira F: Standardization of allergen products: 1 . Detailed characterization of GMP-produced recombinant Bet $\mathrm{v}$ 1.0101 as biological reference preparation. Allergy 2009;64:1038-1045.

11 Michelet L, Lefebvre-Legendre L, Burr SE, Rochaix JD, Goldschmidt-Clermont M: Enhanced chloroplast transgene expression in a nuclear mutant of Chlamydomonas. Plant Biotechnol J 2011;9:565-574.

12 Harris E: The Chlamydomonas Sourcebook, ed 2. Amsterdam, Elsevier, 2008.

13 Sueoka N: Mitotic replication of deoxyribonucleic acid in Chlamydomonas reinhardi. Proc Natl Acad Sci USA 1960;46:83-91.

14 Laffer S, Vangelista L, Steinberger P, Kraft D, Pastore A, Valenta R: Molecular characterization of Bip 1, a monoclonal antibody that modulates IgE binding to birch pollen allergen, Bet v 1. J Immunol 1996;157:4953-4962.
15 Akkerdaas JH, van Ree R, Aalbers M, Stapel SO, Aalberse RC: Multiplicity of cross-reactive epitopes on Bet $\mathrm{v}$ I as detected with monoclonal antibodies and human IgE. Allergy 1995;50:215-220.

16 Almaraz-Delgado AL, Flores-Uribe J, PerezEspana VH, Salgado-Manjarrez E, BadilloCorona JA: Production of therapeutic proteins in the chloroplast of Chlamydomonas reinhardtii. AMB Express 2014;4:57.

17 Kyriakopoulos S, Kontoravdi C: Analysis of the landscape of biologically-derived pharmaceuticals in Europe: dominant production systems, molecule types on the rise and approval trends. Eur J Pharm Sci 2013;48:428441.

18 Rosales-Mendoza S: Future directions for the development of Chlamydomonas-based vaccines. Expert Rev Vaccines 2013;12:10111019.

19 Gregory JA, Shepley-McTaggart A, Umpierrez M, Hurlburt BK, Maleki SJ, Sampson HA, Mayfield SP, Berin MC: Immunotherapy using algal-produced Ara h 1 core domain suppresses peanut allergy in mice. Plant Biotechnol J 2016;14:1541-1550. 